\title{
A Face Recognition Method Based on LBP and GMM
}

\author{
Yuwen Song ${ }^{1}$, Qingling Zhang ${ }^{2,3,{ }^{*}}$ and Xiuquan $\mathrm{Xia}^{1}$ \\ ${ }^{1}$ College of Sciences, Northeastern University, Shenyang, 110819, China \\ ${ }^{2}$ Institute of Systems Science, Northeastern University, Shenyang, 110819, China \\ ${ }^{3}$ State Key Laboratory of Synthetical Automation for Process Industries, Northeastern University, Shenyang, 110819, China \\ ${ }^{*}$ Corresponding author
}

\begin{abstract}
This paper proposes a face recognition method based on local binary pattern(LBP) and Gaussian mixture model(GMM). Firstly, combine Uniform Pattern and Rotation Invariant LBP with traditional LBP operator to obtain initial classification data. Then, adopt GMM to classify face textures, and use EM algorithm to estimate the model parameters where $\mathrm{K}$-means method is applied for initialization. Finally, the experiment is carried out on Yale and ORL face database. The results show that the recognition accuracy of this method has been greatly improved comparing with LBP, PCA or PCA+FLDA alone, especially for small samples.
\end{abstract}

Keywords-face recognition; LBP; GMM; EM algorithm

\section{INTRODUCTION}

With the rapid development of Internet application technology, the continuous expansion of both human virtual and physical activity space, people's demands for information confidentiality and security is increasing. At the same time, there are more and more occasions where people need to perform recognition. Face features are 'easily collected, generally various, chronically stable' for which face recognition technology is one of the most important research topics in the field of human biometric identification.

The theoretical research of face recognition began in 1888 when Calton published an article about human faces identification in Nature ${ }^{[1]}$, but it was not possible to involve automatic recognition of human faces at that time. In 1965, Chan and Bledsoe published a technical report at Panoramic Research $\mathrm{Inc}^{[2]}$. Since then, the prelude of research on automatic face recognition systems has been unveiled. After decades of development, domestic and foreign research institutions have actively carried out research on face recognition technology and obtained abundant accomplishment. In the early 1990s, Turk proposed a method based on Principal Component Analysis(PCA) ${ }^{[3]}$ to extract principal component from high-dimensional data of a face image and ensured that the error of feature reconstruction was the smallest. In 1997, Wiskott et al. proposed Elastic Matching Algorithm ${ }^{[4]}$ which adopts layered structure when constructing the topological map of a human face. The method is more in line with the human face morphological feature, but it has a large amount of computation. In 2004, Ahonen et al. proposed a face expression method based on local texture feature of an image and obtained the beat recognition result on FERET database ${ }^{[5-6]}$. In 2008,
Zhao et al. combined LBP with Gabor and achieved better result in facial expression recognition ${ }^{[7]}$. A face not only has many important local texture features, but also has global structural characteristics. The significant limitation of traditional LBP operator is that LBP codes in adjacent areas are related to their neighboring points, not independent. GMM is a linear combination of multiple Gaussian distribution functions that can theoretically fit any type of distribution.

Therefore, this paper proposes a method based on LBP and GMM. LBP features have significant advantages such as illumination insensitivity, rotation invariance and grayscale invariance, which can describe local detailed texture features well ${ }^{[8]}$. GMM can fit distribution of LBP features, which compensates for the disadvantage that the extracted codes are independent.

\section{LBP OPERATOR THEORY}

The main idea of LBP operator is to set gray value of the center pixel as a threshold; then use this pixel as a center point, $\mathrm{R}$ as a circle radius and assume the circular area containing $\mathrm{P}$ pixels; next compare pixels in circular neighborhood with the threshold successively and obtain binary code used to update the pixel value of the center position. The pixel value can express local texture features.

LBP rotation invariant uniform mode was defined as following ${ }^{[9-10]}$ :

$$
\begin{gathered}
L B P_{P, R}^{r i u 2}=\left\{\begin{array}{cl}
\sum_{i=0}^{P-1} s\left(g_{i}-g_{c}\right) & U\left(L B P_{P, R}\right) \leq 2 \\
P+1 & \text { else }
\end{array}\right. \\
s(x)= \begin{cases}1 & x>0 \\
0 & x \leq 0\end{cases} \\
U\left(L B P_{P, R}\right)=\left|s\left(g_{P-1}-g_{c}\right)-s\left(g_{0}-g_{c}\right)\right|+\sum_{i=1}^{P-1}\left|s\left(g_{i}-g_{c}\right)-s\left(g_{i-1}-g_{c}\right)\right|
\end{gathered}
$$

where:

$g_{c}$ represents gray value of center point at local region 
$g_{p}(p=0,1, \ldots, P)$ is equidistantly distributed points around the central point

\section{THE EsTABLISHMENT OF GMM}

The GMM probability density can be expressed as:

$$
p(x \mid \theta)=\sum_{k=1}^{K} \alpha_{k} f_{k}\left(x \mid \mu_{k}, \Sigma_{k}\right)
$$

where:

$K$ is model order

$\alpha_{k}$ is weighting coefficient, satisfying (2)

$f_{k}$ is the probability density function of the $k^{\text {th }}$ Gaussian component which is shown as (3)

$\mu_{k}$ and $\Sigma_{k}$ is expectation and variance of $f_{k}$

$$
\begin{gathered}
\sum_{k=1}^{K} \alpha_{k}=1 \\
f_{k}\left(x \mid \mu_{k}, \Sigma_{k}\right)=\frac{1}{(2 \pi)^{d / 2}\left|\Sigma_{k}\right|^{1 / 2}} e^{-\frac{1}{2}\left(x-\mu_{k}\right)^{T} \Sigma_{k}^{-1}\left(x-\mu_{k}\right)}
\end{gathered}
$$

Known probability density function for mixed Gaussian model is shown as (1), estimate GMM parameters following the steps of EM algorithm.

\section{E Step $^{[11-13]}$ :}

The expectation of a complete likelihood function is:

$$
\begin{aligned}
Q\left(\theta, \theta^{t-1}\right) & =\sum_{i=1}^{N} \sum_{k=1}^{K} \ln \left(\alpha_{k} f_{k}\left(x \mid \mu_{k}, \Sigma_{k}\right)\right) p\left(k \mid x, \theta^{t-1}\right) \\
& =\sum_{i=1}^{N} \sum_{k=1}^{K} \ln \left(\alpha_{k}\right) p\left(k \mid x, \theta^{t-1}\right)+\sum_{i=1}^{N} \sum_{k=1}^{K} \ln \left(f_{k}\left(x \mid \mu_{k}, \Sigma_{k}\right)\right) p\left(k \mid x, \theta^{t-1}\right)
\end{aligned}
$$

M Steps ${ }^{[14-16]}$ :

$$
\begin{gathered}
\alpha_{k}=\frac{N_{k}^{t}}{N} \quad N_{k}^{t}=\sum_{i=1}^{N} p\left(k \mid x, \theta^{t-1}\right) \\
\mu_{k}=\frac{\sum_{i=1}^{N} x p\left(k \mid x, \theta^{t-1}\right)}{\sum_{i=1}^{N} p\left(k \mid x, \theta^{t-1}\right)}
\end{gathered}
$$

$$
\Sigma_{k}=\frac{\sum_{i=1}^{N} p\left(k \mid x, \theta^{t-1}\right)\left(\Sigma_{k}-\left(x-\mu_{k}\right)\left(x-\mu_{k}\right)^{T}\right)}{\sum_{i=1}^{N} p\left(k \mid x, \theta^{t-1}\right)}
$$

After initializing parameters, the final parameters of GMM can be obtained according to iteration formulas (4)(5)(6). Thus, the complete GMM is established.

\section{LBP-GMM FACE RECOGNITION}

\section{A. Feature Extraction Based on LBP}

In order to improve long connected histograms and low computational efficiency, this paper uses LBP operator including 59 binary modes to encode images, which can reduce data redundancy, decrease the impact of high-frequency noise, and improve program execution speed.

Figure 1 shows original images, their LBP feature maps and statistical histogram of the maps.

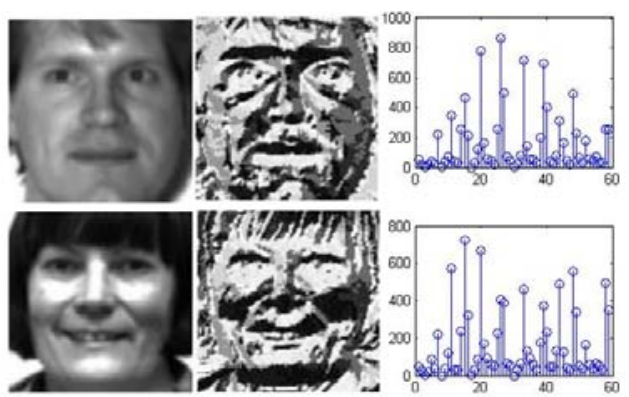

FIGURE I. COMPARISON BETWEEN BEFORE AND AFTER LBP

\section{B. Solve GMM Parameters}

Taking LBP feature extracted in A as original data, this paper adopts K-means method to roughly classify images in the database to obtain initial parameters of the GMM: $\alpha_{0}, \mu_{0}, \Sigma_{0}$.

The basic steps of K-means algorithm are as following:

1.Choose $\mathrm{k}$ arbitrarily from $\mathrm{n}$ data objects as the initial clustering center, where $\mathrm{k}$ is the number of categories;

2.According to each central object, calculate the distance between each object and these central objects, re-distribute the corresponding objects in terms of the minimum distance;

3.Recalculate the central object of each cluster that has changed;

4.When the accuracy is satisfied, the algorithm terminates; otherwise it returns to 2 .

According to formula (4)(5)(6), iterate $\alpha_{0}, \mu_{0}, \Sigma_{0}$ until $\alpha_{k}, \mu_{k}, \Sigma_{k}$ satisfy accuracy. In this paper, the accuracy is equal to $10^{-15}$. Use the above results to classify samples: calculate $f_{1}\left(x \mid \mu_{1}, \Sigma_{1}\right), f_{2}\left(x \mid \mu_{2}, \Sigma_{2}\right), \ldots, f_{k}\left(x \mid \mu_{k}, \Sigma_{k}\right)$ and compare their numeric value. The larger the value is, the greater the probability that the test sample belongs to the component class is. The test sample is assigned to the largest component class. 
The basic steps of LBP-GMM algorithm are as following:

1: Traverse the database to extract LBP features of each image;

2: The model order is known. K-means algorithm is used to roughly classify the new data gotten in Step1 to obtain the initial values of the model parameters;

3: Use EM algorithm to estimate parameter, iterate them until meet accuracy conditions and output the final parameter results to complete the establishment of GMM;

4: Bring LBP eigenvalues of the sample image into each component of GMM, calculate the size and output the classification result.

\section{EXPERIMENTAL RESULTS AND ANALYSIS}

This experiment was tested on Yale face database and ORL face database. Yale database contains 165 images of 15 individuals. These images contain changes in lighting, expression and posture. The dimension is $100 * 100$. ORL database contains 40 individuals, each 10 images, including changes in expression, pose and scale. The size of it is $92 * 112$. In the experiment, each of 1,3 and 5 images were used as a training sample and the rest of images were used as test samples.

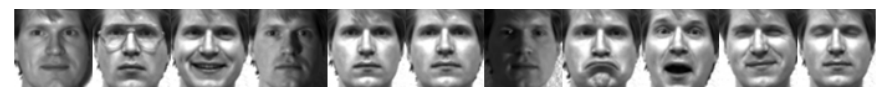

FIGURE II. EXAMPLES OF FACE IMAGES IN YALE FACE DATABASE

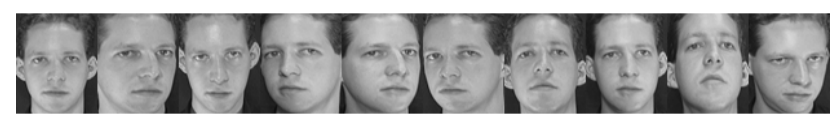

FIGURE III. EXAMPLES OF FACE IMAGES IN ORL FACE DATABASE

The experimental results are shown in Table I and Table II.

TABLE I. COMPARISON OF DIFFERENT ALGORITHMS ON YALE FACE DETABASE

\begin{tabular}{|c|c|c|c|c|}
\hline \multirow{2}{*}{$\begin{array}{c}\text { Train } \\
\text { number }\end{array}$} & \multicolumn{4}{|c|}{ Recognition rate } \\
\cline { 2 - 5 } & LBP & PCA & PCA+FLDA & LBP-GMM \\
\hline 1 & $58 \%$ & $44 \%$ & & $62 \%$ \\
\hline 3 & $83 \%$ & $83 \%$ & $85 \%$ & $88 \%$ \\
\hline 5 & $84 \%$ & $83 \%$ & $83 \%$ & $89 \%$ \\
\hline
\end{tabular}

TABLE II. COMPARISON OF DIFFERENT ALGORITHMS ON ORL FACE DETABASE

\begin{tabular}{|c|c|c|c|c|}
\hline \multirow{2}{*}{$\begin{array}{c}\text { Train } \\
\text { number }\end{array}$} & \multicolumn{4}{|c|}{ Recognition rate } \\
\cline { 2 - 5 } & $\mathbf{L B P}$ & $\boldsymbol{P C A}$ & $\boldsymbol{P C A}+\boldsymbol{F L D A}$ & LBP-GMM \\
\hline 1 & $73 \%$ & $71 \%$ & & $77 \%$ \\
\hline 3 & $82 \%$ & $85 \%$ & $84 \%$ & $87 \%$ \\
\hline 5 & $84 \%$ & $84 \%$ & $85 \%$ & $89 \%$ \\
\hline
\end{tabular}

As can be seen from Table I and Table II, the algorithm in this paper has achieved a higher recognition rate in the Yale face database and ORL face database. In the case of a small number of training samples (such as one or three), the traditional method of PCA, FLDA and LBP can only achieve low recognition rate or failure because of the influence of expression and posture. However, the recognition rate obtained by this method is obviously higher than other method, which has important significance for small sample face recognition.

\section{CONCLUSION}

Rotation-invariant LBP operator is robust to gray scale while GMM can fit probability distribution of any shape. EM algorithm is an effective way to solve distribution parameters from 'incomplete data'. This lays a foundation for the application of LBP-GMM face recognition in this paper. Experiments show that recognition rate of this algorithm in small samples is obviously higher than that of traditional LBP, PCA and PCA+FLDA.

For EM estimation, the most important issue is how to set initial parameters. In the discussion of this paper, K-means is used to initialize parameters. However, in practical applications, the estimation accuracy and computing efficiency should be taken into consideration when initializing parameters in order to improve algorithm accuracy. It's the problem to be studied later.

\section{REFERENCES}

[1] Feifei Huang. The face recognition study based on LBP[D].Chongqing University

[2] H. Chan, W.W.Bledsoe. A man-machine facial recognition system: some preliminary results[R]. Technical report, Panoramic Research Inc., Cal, 1965. Girish G.N, Shrinivasa Naika C.L,Das P.K. Face recognition using MB-LBP and PCA: A comparative study[C]. International Conference on Computer Communication and Informatics, 2014:2-6.

[3] Chao Liu.Face Verification and Recognition System based on Elastic Graph Matching[D]. Harbin: Harbin Institute of Technology, 2006:5-7.

[4] AHONEN T, HADID A, PIETIKAINEN M. Face recognition with local binary patterns[C]//ECCv. $8^{\text {th }}$ European Conference on Computer Vision. Prague: ECCV, 2004: 469-481.

[5] AHONEN T, HADID A, PIETIKAINEN M. Face description with local binary patterns: Application to face recognition[J]. IEEE Transactions on Pattern Analysis and Machine Intelligence, 2006,28(12):2037-2041.

[6] TANG H L, SUN Y F, YIN B C, et al. Face recognition based on Haar LBP histogram[J]. ICACTE, 2010, 6:235-238.

[7] Xian Wang,Yan Zhang, Xin Mu. A Face Recognition Algorithm based on improved LBP[J]. Electro-Optics Engineering. 2012( 7) : 109 -114.

[8] Ojala T, Pietikainen M \& Maenpaa T. A generalized Local Binary Pattern pperator for multiresolution gray scale and rotation invariant texture classification[J]. Second International Conference on Advances in Pattern Recognition, Rio de Janeiro, Brazil,2001:397-406.

[9] Ling Zhang,Yi min Cheng,Yuming Xie,Jie Li.Video Object Shadow Detection Method based on Local Binary Image[J].Systems Engineering and Electronics,2007, 29(6):974-977.

[10] Zhirong Ji, Keming Huang. Parameter Estimation based on Noninvalidated Data. Journal of Fuzhou University (JCR-SCI), 2007,35(4):507-510.

[11] Xiangyun Zhang, Sishui Wang. Parameter Estimation based on Gaussian Mixture Density[J]. J.Hangzhou Teac.Coll.Nat.Sci.Ed, 2005,4(5):349352.

[12] Jinqin Zhong, Lichuan Gu, Jieqing Tan, Yingying Li. Parameter Estimation based on Split Algorithm[J]. Computer Engineering and Application, 2012, 48(34):28-32. 
[13] A P Dempster, N M laired, D B Rubin. Maximum likelihood from incomplete data via the EM algorithm[J]. Journal of the Royal Statistical Society, 1977, B(39):1-38.

[14] Gyemin Lee, Clayton Scott. EM algorithms for multivariate Gaussian mixture models with truncated and censored data[J]. Computational Statistics \& Analysis, 56(9):2816-2829.

[15] Christophe Biernackia, Gilles Celeuxb, Gerard Govaertc. Choosing starting values for the EM algorithm for getting the highest likelihood in multivariate Gaussian texture models[J]. Computational Statistics \& Analysis, 2003, 41(3-4):561-575. 